\title{
Comparison of survival outcomes of locally advanced breast cancer patients receiving post- mastectomy radiotherapy with and without immediate breast reconstruction: a population- based analysis
}

This article was published in the following Dove Press journal: Cancer Management and Research

\author{
San-Gang $\mathrm{Wu}^{1}{ }^{1, *}$ \\ Wen-Wen Zhang',* \\ Jia-Yuan Sun ${ }^{2}$ \\ Qin Lin' \\ Zhen-Yu $\mathrm{He}^{2}$ \\ 'Department of Radiation Oncology, \\ Xiamen Cancer Hospital, The First \\ Affiliated Hospital of Xiamen \\ University, Xiamen 361003, People's \\ Republic of China; ${ }^{2}$ Department of \\ Radiation Oncology, Sun Yat-Sen \\ University Cancer Center, State Key \\ Laboratory of Oncology in South \\ China, Collaborative Innovation \\ Center of Cancer Medicine, \\ Guangzhou 5I0060, People's Republic \\ of China
}

*These authors contributed equally to this work

Correspondence: Qin Lin Department of Radiation Oncology, Xiamen Cancer Hospital, the First Affiliated Hospital of Xiamen University, 55 Zhenhai Road, Xiamen 361003,

People's Republic of China

Tel +86592 2139531

Fax +86592 213 7301

Email linqin953।@।26.com

Zhen-Yu He

Department of Radiation Oncology, Sun Yat-sen University Cancer Center, State Key Laboratory of Oncology in South China, Collaborative Innovation Center of Cancer Medicine, 65I Dongfeng Road East, Guangzhou 510060, People's Republic of China

Tel +86 2087343543

Fax +862087343392

Email hezhy@sysucc.org.cn
Objective: To compare the survival outcomes in locally advanced breast cancer (LABC) patients receiving post-mastectomy radiotherapy (PMRT) with and without immediate breast reconstruction.

Methods: We used the Surveillance, Epidemiology, and End Results program to include LABC patients who were treated/not treated with immediate breast reconstruction followed by PMRT between 2003 and 2010. Statistical analysis was performed using the chi-squared test, Kaplan-Meier survival analysis, and Cox regression analysis. A 1:1 propensity score matching method was performed to decrease the selection bias.

Results: We identified 1,732 patient-pairs that were completely matched. In the unmatched population, 8,198 and 1,802 patients received mastectomy only and immediate breast reconstruction, respectively. Patients who received immediate breast reconstruction had better breast cancer-specific survival (BCSS) (hazard ratio [HR] 0.880, 95\% CI 0.783-0.989, $P=$ 0.032 ) and overall survival (OS) (HR 0.846, 95\% CI 0.758-0.943, $P=0.003$ ) than patients who underwent mastectomy alone. However, in the matched population, there was comparable BCSS and OS between patients who received immediate breast reconstruction and mastectomy alone. Subset analysis in the matched population found that immediate breast reconstruction was associated with better BCSS (HR 0.750, 95\% CI 0.614-0.917, $P=0.005$ ) and OS (HR $0.779,95 \%$ CI $0.644-0.942, P=0.010)$ compared to patients aged $<50$ years who received mastectomy alone.

Conclusion: There are comparable survival outcomes in LABC patients who received immediate breast reconstruction or mastectomy alone followed by PMRT. However, patients aged $<50$ years had a survival advantage after immediate breast reconstruction.

Keywords: breast cancer, irradiation, autologous tissue, implant, prognosis

\section{Introduction}

Locally advanced breast cancer (LABC) is most commonly defined as stage III disease, and mastectomy and lymphadenectomy are the standard surgical procedures for LABC, while postoperative mastectomy radiotherapy (PMRT) could significantly decrease locoregional recurrence and confer additional survival advantage. ${ }^{1,2}$ Patients who undergo mastectomy deal with impaired sexual characteristics and body image, which may negatively impact the quality of life. These drawbacks may be effectively compensated in some women by breast reconstruction using autologous tissue or implant, especially 
when performed immediately following mastectomy. Patients who received breast reconstruction may then still experience positive self-perception and natural appearance, although complications may affect the cosmetic effects.

Although several studies have indicated that immediate breast reconstruction in breast cancer is both feasible and oncologically safe, PMRT for patients who underwent immediate breast reconstruction may lead to higher rates of adverse events including reconstruction failure, capsular contracture, and overall complications. ${ }^{3-9}$ Patients who received implant reconstruction had a significantly higher incidence of reconstruction failure compared to autologous reconstruction after PMRT. ${ }^{6}$ In addition, in patients who had not experienced reconstruction failure, PMRT after autologous reconstruction was associated with a superior patient-reported satisfaction and a higher psychosocial or physical well-being than implant reconstruction followed by PMRT. ${ }^{3}$ Therefore, patients who are eligible to receive PMRT are less likely to receive immediate breast reconstruction, ${ }^{10}$ and anticipation of PMRT may lead to delay or omission of breast reconstruction. However, attitudes toward breast reconstruction in the setting of planned PMRT are changing. According to the Surveillance, Epidemiology, and End Results (SEER) results program from 2000 to 2010, the proportion of immediate breast reconstruction among patients receiving PMRT increased from $13.6 \%$ to $25.1 \% .{ }^{11}$ However, another SEER study also found that an increase in PMRT was not associated with a decrease in those receiving reconstruction. ${ }^{12}$ There are conflicting results with regard to survival outcomes in LABC patients who receive immediate breast reconstruction followed by PMRT. Some studies have indicated that no statistically significant differences were found in survival outcomes between those receiving breast reconstruction and mastectomy alone. ${ }^{13-15}$ In contrast, a study showed that patients who received breast reconstruction had better breast cancer-specific survival (BCSS) than patients who underwent mastectomy alone. ${ }^{16}$ The heterogeneity of the population and the difference in sample size may be the main reasons for these contrasting results.

Population-based cancer registry includes a wide range of patient demographics and avoids the inherent bias observed in single institution studies, thereby allowing for greater generalization. In the current study, we used the SEER database to determine the clinical characteristics and survival outcomes of patients with LABC who received immediate breast reconstruction or mastectomy alone, followed by PMRT.

\section{Materials and methods}

\section{Patients}

The SEER database is maintained by the National Cancer Institute and includes demographics, incidence, and cancerspecific survival data for $\sim 28 \%$ of the population in the USA. ${ }^{17}$ The present study was based on the publicly available SEER database, and we have obtained the permission to access them with the reference number 11025-Nov 2016. To investigate the effect of immediate breast reconstruction (reconstruction within 4 months of mastectomy as defined by SEER $^{18,19}$ ) on the long-term survival of patients, we included patients diagnosed with breast cancer between 2004 and 2010. Patients who met the following criteria were included: 1) histologically confirmed breast cancer; 2) American Joint Committee on Cancer, sixth edition, tumor-node-metastasis staging system staged $\mathrm{T} 1-4 \mathrm{~N} 2-3 \mathrm{M} 0 ; 3)$ receiving mastectomy alone or immediate breast reconstruction including reconstruction not otherwise specified, tissue, implant, or combined with tissue and implant reconstruction; 4) receiving PMRT (beam radiation) and chemotherapy; 5) variables including age, race/ethnicity, grade, estrogen receptor (ER) status, progesterone receptor (PR) status, and marital status. Patients who received preoperative or intraoperative radiotherapy were excluded. The ethics committee of the First Affiliated Hospital of Xiamen University approved this study.

The following demographic, clinicopathologic, and treatment data were collected: age and year at diagnosis, race/ethnicity, grade, $\mathrm{T}$ stage, $\mathrm{N}$ stage, hormone receptors status, marital status, and reconstruction methods. The primary survival points were BCSS and overall survival (OS). BCSS and OS were defined as the time interval from initial diagnosis until breast cancer-related death or death from any cause, respectively.

\section{Statistical analysis}

The differences in demographic and clinicopathologic variables between immediate breast reconstruction and mastectomy alone were compared using the chi-squared test. To decrease the impact of potential selection bias in the retrospective studies, a one-to-one propensity score matching (PSM) method was performed using the following variables: age, race/ethnicity, grade, $\mathrm{T}$ stage, $\mathrm{N}$ stage, ER status, PR status, and marital status. ${ }^{20,21}$ BCSS and OS were assessed using Kaplan-Meier survival methods and compared with a log-rank test. Cox proportional hazards regression was used to evaluate the variables and potential association with BCSS and OS. Variables with statistical significance by 
univariate analysis were entered into multivariate analysis. Hazard ratios (HRs) with the corresponding 95\% CIs were calculated to assess the risk of death. All statistical analyses were performed using the SPSS software package, version 22.0 (IBM Corporation, Armonk, NY, USA). The level of statistical significance was defined at $P<0.05$.

\section{Results}

\section{Patient characteristics}

We included 10,000 breast cancer patients with stage T1-4N2-3M0 who met the inclusion criteria. These included $8,198(82.0 \%)$ patients receiving mastectomy alone and $1,802(18.0 \%)$ patients who received immediate breast reconstruction. In patients who received immediate breast reconstruction, $664(36.8 \%)$ patients received autologous tissue reconstruction, $655(36.3 \%)$ patients received implant reconstruction, and 483 (26.8\%) patients had reconstruction that was not otherwise specified or combined with tissue and implant reconstruction. The number of patients undergoing immediate breast reconstruction increased from 2004 to 2010 ( $P<0.001$; Figure $1 \mathrm{~A})$. In addition, more patients were likely to receive breast reconstruction with autologous tissue implantation before 2008 (55.9\% vs 44.1\%), while more patients were likely to receive breast reconstruction with implantation in 2009 and 2010 (59.3\% vs 40.7\%) $(P<0.001$; Figure 1B). The patient features of the entire cohort are summarized in Table 1.

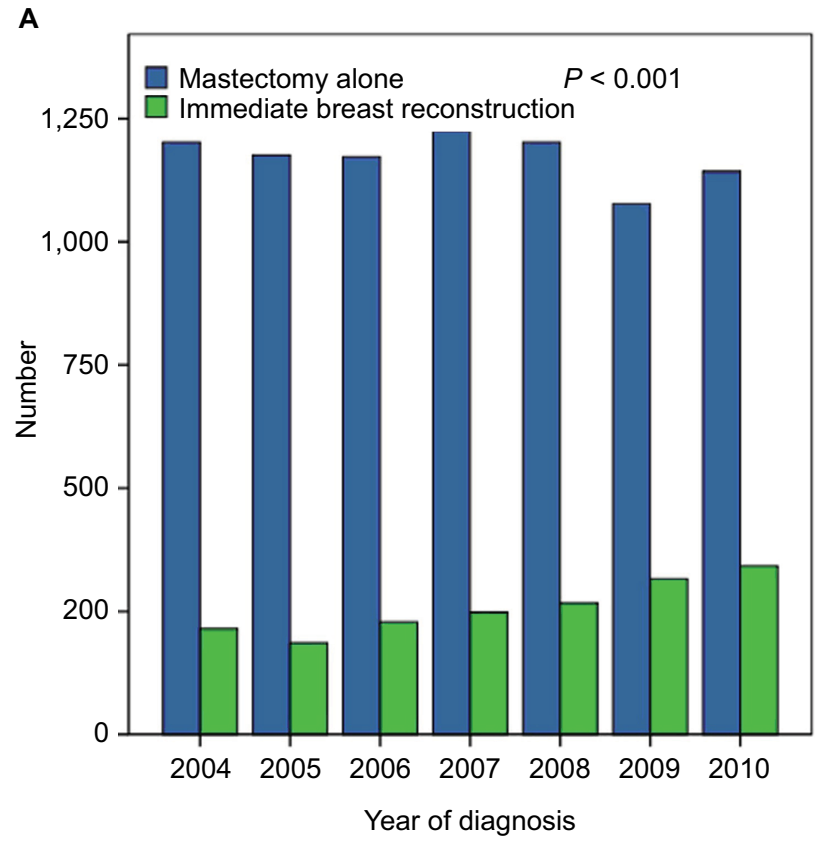

\section{Survival and prognostic analysis in the unmatched population}

The median follow-up period was 61 months (range 3-119 months). The 5-year BCSS and OS were $78.3 \%$ and $74.4 \%$, respectively. The 5-year BCSS in patients who received breast reconstruction and mastectomy alone was $82.4 \%$ and $77.4 \%$, respectively ( $P<0.001$; Figure $2 \mathrm{~A})$, while the 5 -year OS was $80.3 \%$ and $73.1 \%$, respectively $(P<0.001$; Figure $2 \mathrm{~B})$. There was comparable BCSS $(P=0.998)$ and $\mathrm{OS}(P=0.921)$ in patients who received autologous tissue reconstruction or implant reconstruction.

In the univariate and multivariate Cox analyses (Table 2), immediate breast reconstruction was associated with better BCSS (HR 0.880, 95\% CI 0.783-0.989, $P=0.032$ ) and OS (HR 0.846, 95\% CI 0.758-0.943, $P=0.003$ ) compared to patients who underwent mastectomy alone. Age at diagnosis, race/ethnicity, tumor grade, $\mathrm{T}$ stage, $\mathrm{N}$ stage, hormone receptor status, and marital status were also independent prognostic factors included in the multivariate analysis.

\section{Prognostic analysis in the matched population}

A total of 1,732 pairs of patients were completely matched. The patient features of the matched population are listed in Table 1. There were comparable BCSS ( $P=0.267$; Figure $3 \mathrm{~A})$ and $\operatorname{OS}(P=0.313$; Figure $3 \mathrm{~B})$ in patients who received

B

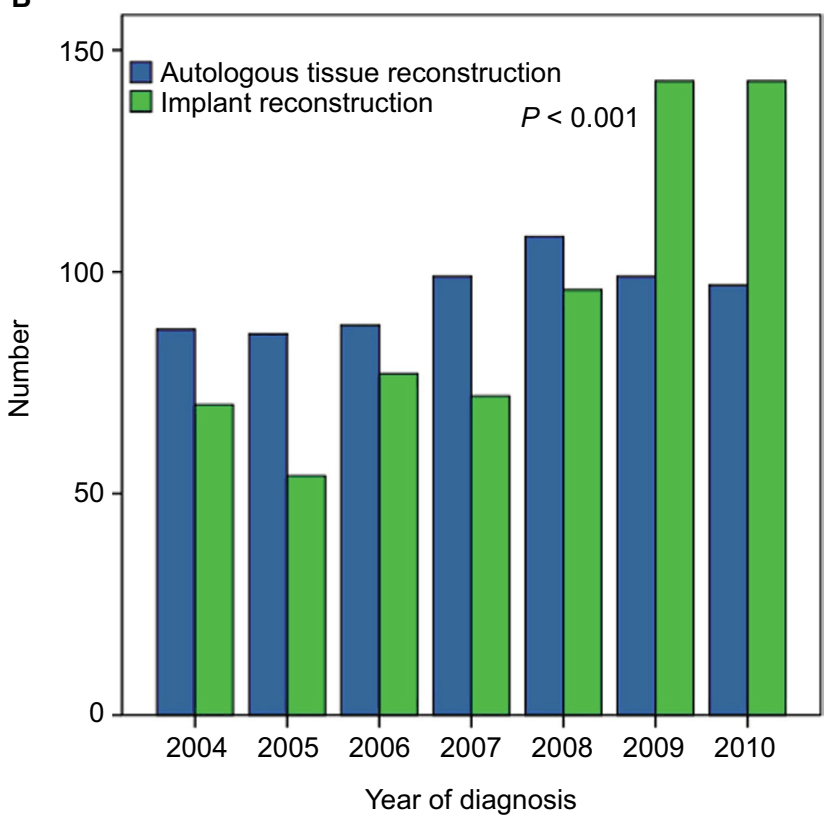

Figure I Frequency of patients with and without immediate breast reconstruction (A) and frequency of patients with autologous tissue reconstruction or implant reconstruction (B), 2003-2010. 
Table I Patient characteristics before and after propensity score matching

\begin{tabular}{|c|c|c|c|c|c|c|c|c|}
\hline \multirow[t]{2}{*}{ Variables } & \multicolumn{4}{|l|}{ Before PSM } & \multicolumn{4}{|c|}{ After PSM } \\
\hline & n (\%) & $\begin{array}{l}\text { Immediate } \\
\text { breast } \\
\text { reconstruction }\end{array}$ & $\begin{array}{l}\text { Mastectomy } \\
\text { alone }\end{array}$ & $P$ & n & $\begin{array}{l}\text { Immediate } \\
\text { breast } \\
\text { reconstruction }\end{array}$ & $\begin{array}{l}\text { Mastectomy } \\
\text { alone }\end{array}$ & $P$ \\
\hline \multicolumn{9}{|l|}{ Age (years) } \\
\hline$<50$ & $3784(37.8)$ & $991(55.0)$ & $2793(34.1)$ & $<0.001$ & 1884 & 942 & 942 & I \\
\hline $50-64$ & $4205(42.1)$ & $691(38.3)$ & $3514(42.9)$ & & 1346 & 673 & 673 & \\
\hline$\geq 65$ & $2011(20.1)$ & $120(6.7)$ & $1891(23.1)$ & & 234 & 117 & 117 & \\
\hline \multicolumn{9}{|l|}{ Race/ethnicity } \\
\hline White & $7943(79.4)$ & I522 (84.5) & $6421(78.3)$ & $<0.001$ & 2986 & 1493 & 1493 & I \\
\hline Black & $1187(11.9)$ & $172(9.5)$ & $1015(12.4)$ & & 292 & 146 & 146 & \\
\hline Other & $870(8.7)$ & $108(6.0)$ & $762(9.3)$ & & 186 & 93 & 93 & \\
\hline \multicolumn{9}{|l|}{ Grade } \\
\hline Well differentiated & $698(7.0)$ & I46 (8.I) & $552(6.7)$ & 0.004 & 246 & 123 & 123 & I \\
\hline Moderately differentiated & $3847(38.5)$ & $732(40.6)$ & $3115(38.0)$ & & 1420 & 710 & 710 & \\
\hline Poorly/undifferentiated & $5455(45.5)$ & $924(5 \mathrm{I} .3)$ & $4531(55.3)$ & & 1798 & 899 & 899 & \\
\hline \multicolumn{9}{|l|}{ Tumor stage } \\
\hline $\mathrm{TI}$ & $1672(16.7)$ & 398 (2I.5) & $1283(15.7)$ & $<0.001$ & 718 & 359 & 359 & I \\
\hline $\mathrm{T} 2$ & $4604(46.0)$ & $860(47.7)$ & 3744 (45.7) & & 1698 & 849 & 849 & \\
\hline T3 & $2336(23.4)$ & $425(23.6)$ & $1911(23.3)$ & & 812 & 406 & 406 & \\
\hline $\mathrm{T} 4$ & 1388 (13.9) & $128(7.1)$ & $1260(15.4)$ & & 236 & 118 & 118 & \\
\hline \multicolumn{9}{|l|}{ Nodal stage } \\
\hline N2 & $6364(63.6)$ & $1217(67.5)$ & $5147(62.8)$ & $<0.001$ & 2368 & 1184 & 1184 & I \\
\hline N3 & $3636(36.4)$ & $585(32.5)$ & $305 I(37.2)$ & & 1096 & 548 & 548 & \\
\hline \multicolumn{9}{|l|}{ ER } \\
\hline Negative & 2794 (27.9) & $432(24.0)$ & $2362(28.8)$ & $<0.001$ & 820 & 410 & 410 & I \\
\hline Positive & 7206 (72.1) & $1370(76.0)$ & $5836(7 \mid .2)$ & & 2644 & 1322 & 1322 & \\
\hline \multicolumn{9}{|l|}{ PR } \\
\hline Negative & $4064(40.6)$ & $627(34.8)$ & 3437 (4I.9) & $<0.001$ & 1194 & 597 & 597 & I \\
\hline Positive & $5936(59.4)$ & 1175 (65.2) & $476 I(58.1)$ & & 2270 & 1135 & 1135 & \\
\hline \multicolumn{9}{|l|}{ Marital status } \\
\hline Married & $6192(61.9)$ & I 248 (69.3) & $4944(60.3)$ & $<0.001$ & 2430 & 1215 & 1215 & I \\
\hline Unmarried & $3808(38.1)$ & $554(30.7)$ & 3254 (39.7) & & 1034 & 517 & 517 & \\
\hline
\end{tabular}

Abbreviations: ER, estrogen receptor; HR, hazard ratio; N, node; PR, progesterone receptor; PSM, propensity score matching; T, tumor.

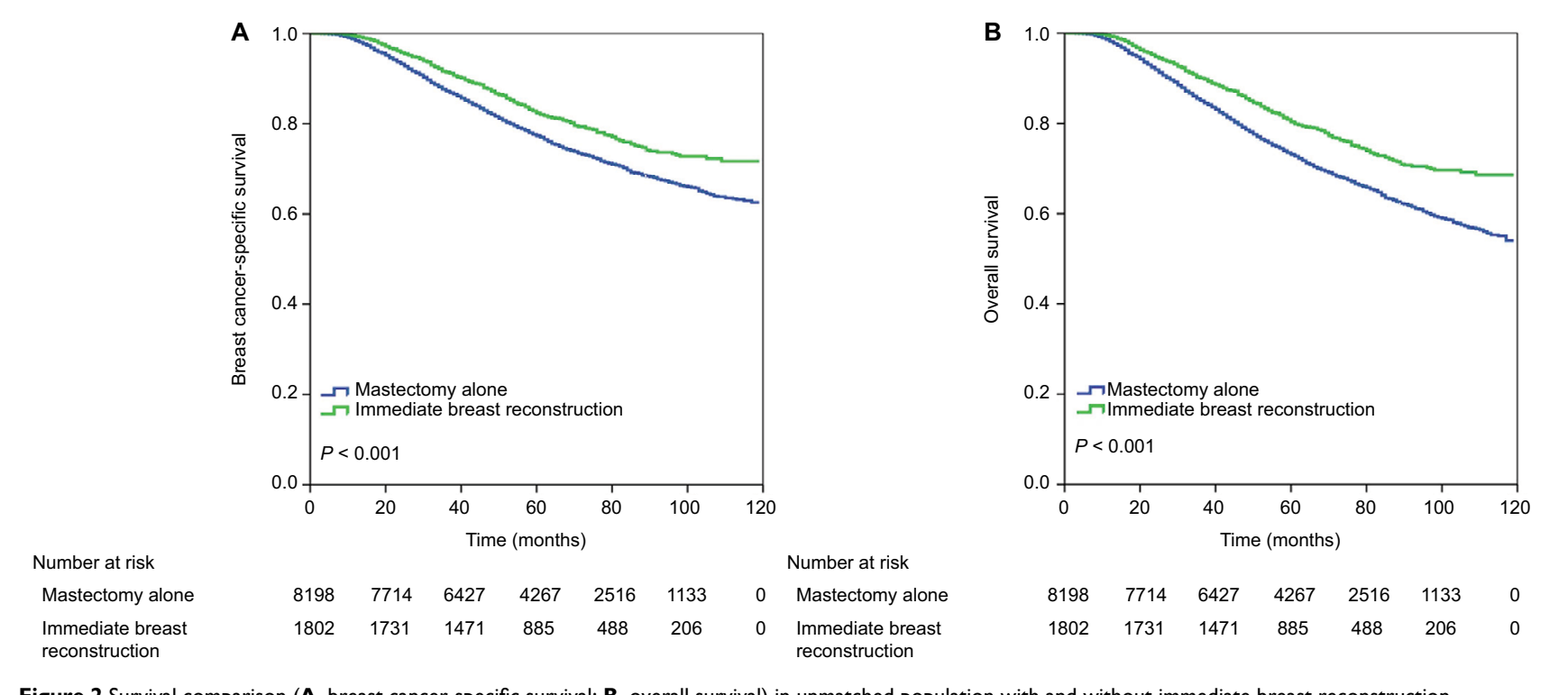

Figure 2 Survival comparison (A, breast cancer-specific survival; B, overall survival) in unmatched population with and without immediate breast reconstruction. 
Table 2 Univariate and multivariate Cox regression analyses of prognostic factors before propensity score matching

\begin{tabular}{|c|c|c|c|c|c|c|c|c|c|c|c|c|}
\hline \multirow[t]{3}{*}{ Variables } & \multicolumn{6}{|c|}{ Univariate } & \multicolumn{6}{|c|}{ Multivariate } \\
\hline & \multicolumn{3}{|c|}{ BCSS } & \multicolumn{3}{|l|}{ OS } & \multicolumn{3}{|c|}{ BCSS } & \multicolumn{3}{|l|}{ OS } \\
\hline & HR & $95 \% \mathrm{Cl}$ & $P$ & HR & $95 \% \mathrm{Cl}$ & $P$ & HR & $95 \% \mathrm{Cl}$ & $P$ & HR & $95 \% \mathrm{Cl}$ & $P$ \\
\hline \multicolumn{13}{|l|}{ Age (years) } \\
\hline$<50$ & I & & & 1 & & & 1 & & & I & & \\
\hline $50-64$ & 1.041 & $0.952-1.138$ & 0.381 & 1.126 & $1.036-1.224$ & 0.005 & 0.977 & $0.892-1.069$ & 0.609 & 1.059 & $0.974-1.152$ & 0.18 \\
\hline$\geq 65$ & 1.234 & 1.109-1.372 & $<0.001$ & 1.588 & I.447-I.743 & $<0.001$ & 1.184 & $1.06 \mathrm{I}-1.320$ & 0.003 & $1.5 \mathrm{I}$ & $1.372-1.663$ & $<0.001$ \\
\hline \multicolumn{13}{|l|}{ Race/ethnicity } \\
\hline White & I & & & I & & & I & & & 1 & & \\
\hline Black & 1.484 & $1.329-1.658$ & $<0.001$ & 1.542 & $|.397-1.70|$ & $<0.001$ & 1.291 & $1.153-1.446$ & $<0.001$ & 1.366 & $1.235-1.511$ & $<0.001$ \\
\hline Other & 0.901 & $0.776-1.046$ & 0.17 & 0.88 & $0.767-1.009$ & 0.066 & 0.836 & $0.720-0.97 \mid$ & 0.019 & 0.833 & $0.726-0.953$ & 0.009 \\
\hline \multicolumn{13}{|l|}{ Grade } \\
\hline Well differentiated & I & & & I & & & I & & & 1 & & \\
\hline $\begin{array}{l}\text { Moderately } \\
\text { differentiated }\end{array}$ & 1.688 & $1.338-2.219$ & $<0.001$ & 1.468 & $1.209-1.782$ & $<0.001$ & 1.556 & $1.233-1.963$ & $<0.001$ & 1.379 & $1.135-1.675$ & 0.001 \\
\hline $\begin{array}{l}\text { Poorly/ } \\
\text { undifferentiated }\end{array}$ & 3.033 & $2.420-3.801$ & $<0.001$ & 2.486 & $2.060-3.000$ & $<0.001$ & 2.08 & $1.652-2.619$ & $<0.001$ & 1.795 & $1.480-2.175$ & $<0.001$ \\
\hline \multicolumn{13}{|l|}{ Tumor stage } \\
\hline TI & I & & & I & & & 1 & & & I & & \\
\hline $\mathrm{T} 2$ & 1.531 & $1.332-1.760$ & $<0.001$ & 1.466 & $1.297-1.656$ & $<0.001$ & 1.459 & $1.269-1.678$ & $<0.001$ & 1.403 & $1.242-1.586$ & $<0.001$ \\
\hline T3 & 2.137 & $1.847-2.472$ & $<0.001$ & 1.926 & $1.692-2.192$ & $<0.001$ & 2.112 & I.824-2.446 & $<0.001$ & 1.927 & $1.692-2.195$ & $<0.001$ \\
\hline $\mathrm{T} 4$ & 3.469 & $2.989-4.026$ & $<0.001$ & 3.141 & $2.752-3.585$ & $<0.001$ & 2.779 & $2.391-3.231$ & $<0.001$ & 2.536 & $2.219-2.898$ & $<0.001$ \\
\hline \multicolumn{13}{|l|}{ Nodal stage } \\
\hline N2 & I & & & I & & & I & & & I & & \\
\hline N3 & 1.802 & $1.665-1.950$ & $<0.001$ & 1.707 & $1.589-1.834$ & $<0.001$ & 1.585 & $1.463-1.716$ & $<0.001$ & 1.506 & $1.404-1.619$ & $<0.001$ \\
\hline \multicolumn{13}{|l|}{ ER } \\
\hline Negative & I & & & 1 & & & $\mathrm{I}$ & & & I & & \\
\hline Positive & 0.417 & $0.385-0.45 I$ & $<0.001$ & 0.444 & $0.413-0.478$ & $<0.001$ & 0.66 & $0.589-0.740$ & $<0.001$ & 0.666 & $0.600-0.738$ & $<0.001$ \\
\hline \multicolumn{13}{|l|}{ PR } \\
\hline Negative & I & & & I & & & 1 & & & 1 & & \\
\hline Positive & 0.446 & $0.412-0.483$ & $<0.001$ & 0.478 & $0.445-0.513$ & $<0.001$ & 0.686 & $0.614-0.767$ & $<0.001$ & 0.726 & $0.656-0.802$ & $<0.001$ \\
\hline \multicolumn{13}{|l|}{ Marital status } \\
\hline Married & I & & & 1 & & & I & & & I & & \\
\hline Unmarried & 0.758 & $0.700-0.821$ & $<0.001$ & 0.722 & $0.672-0.776$ & $<0.001$ & 0.859 & $0.792-0.933$ & $<0.001$ & 0.838 & $0.778-0.902$ & $<0.001$ \\
\hline \multicolumn{13}{|c|}{ Immediate breast reconstruction } \\
\hline No & $\mathrm{I}$ & & & I & & & I & & & I & & \\
\hline Yes & 0.731 & $0.652-0.819$ & $<0.001$ & 0.677 & $0.609-0.753$ & $<0.001$ & 0.88 & $0.783-0.989$ & 0.032 & 0.846 & $0.758-0.943$ & 0.003 \\
\hline
\end{tabular}

Abbreviations: BCSS, breast cancer-specific survival; ER, estrogen receptor; HR, hazard ratio; N, node; OS, overall survival; PR, progesterone receptor; T, tumor.

breast reconstruction and mastectomy alone in the matched population.

The results of univariate analyses showed that immediate breast reconstruction was not associated with a better BCSS (HR $1.088,95 \%$ CI $0.937-1.2629, P=0.268$ ) or OS (HR $1.074,95 \% \mathrm{CI} 0.935-1.235, P=0.314$ ) compared to patients who underwent mastectomy alone (Table 3 ). Tumor grade, T stage, $\mathrm{N}$ stage, hormone receptor status, and marital status were also independent prognostic factors in the multivariate analysis of the matched population (Table 3).

\section{Subset analysis in the matched population}

We further performed a subset analysis in the matched population, and the results showed that breast reconstruction had no effect on BCSS and OS for all the demographic and clinicopathologic variables, except in patients aged $<50$ years. In Kaplan-Meier survival analysis, the BCSS $(P=0.012$; Figure 4A) and OS $(P=0.023$; Figure $4 \mathrm{~B})$ were better in those patients who underwent immediate breast reconstruction compared to patients who received mastectomy alone.

After adjustment by race/ethnicity, tumor grade, $T$ stage, $\mathrm{N}$ stage, hormone receptor status, and marital status, patients who received immediate breast reconstruction still had better BCSS (HR 0.750, 95\% CI 0.614-0.917, $P=0.005$ ) and OS (HR 0.779, 95\% CI 0.644-0.942, $P=0.010$ ) than those treated with mastectomy alone (Table 4).

\section{Discussion}

In this study, we explored the effect of immediate breast reconstruction on LABC patients after PMRT. Our results 


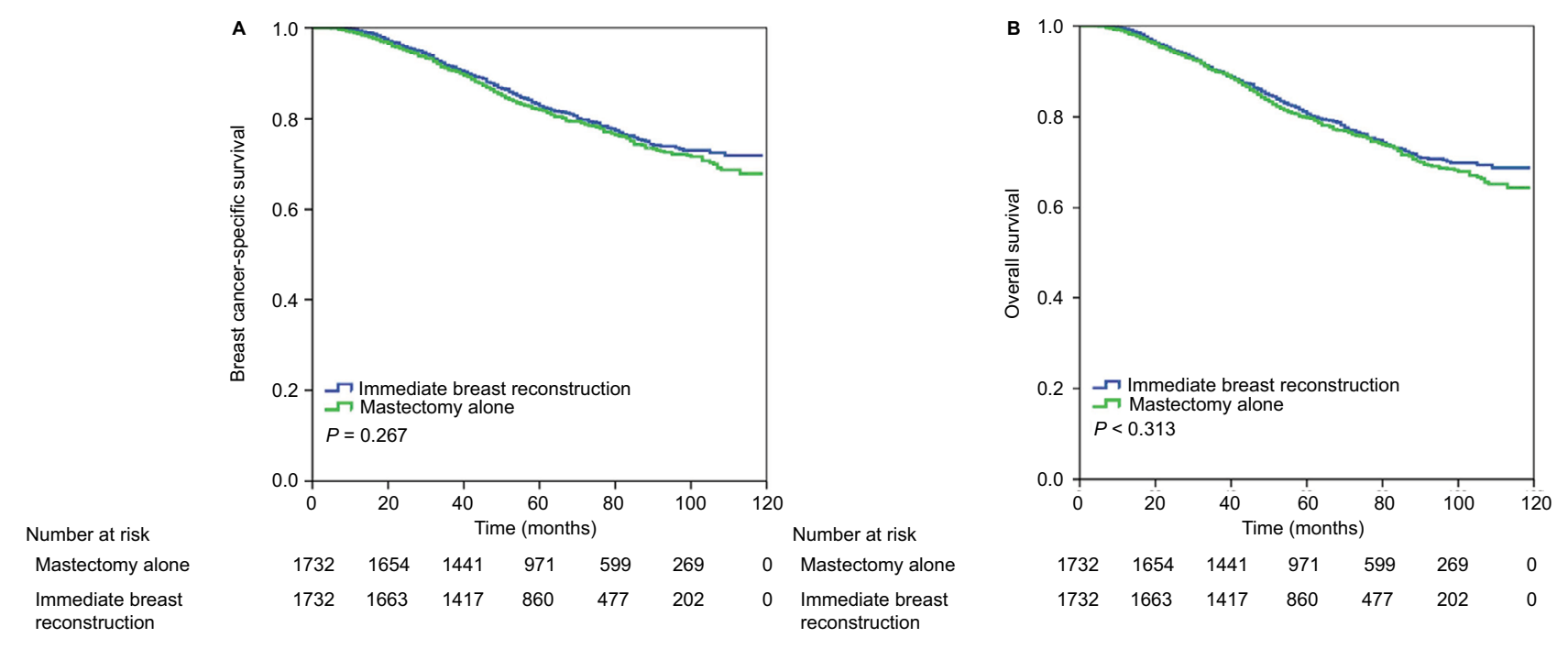

Figure 3 Survival comparison (A, breast cancer-specific survival; B, overall survival) in matched population with and without immediate breast reconstruction.

Table 3 Univariate and multivariate Cox regression analyses of prognostic factors after propensity score matching

\begin{tabular}{|c|c|c|c|c|c|c|c|c|c|c|c|c|}
\hline \multirow[t]{3}{*}{ Variables } & \multicolumn{6}{|c|}{ Univariate } & \multicolumn{6}{|c|}{ Multivariate } \\
\hline & \multicolumn{3}{|c|}{ BCSS } & \multicolumn{3}{|l|}{ OS } & \multicolumn{3}{|l|}{ BCSS } & \multicolumn{3}{|l|}{ OS } \\
\hline & HR & $95 \% \mathrm{Cl}$ & $P$ & HR & $95 \% \mathrm{Cl}$ & $P$ & HR & $95 \% \mathrm{Cl}$ & $P$ & HR & $95 \% \mathrm{Cl}$ & $P$ \\
\hline \multicolumn{13}{|l|}{ Age (years) } \\
\hline$<50$ & I & & & I & & & 1 & & & - & & \\
\hline $50-64$ & 1.031 & $0.884-1.203$ & 0.698 & 1.073 & $0.928-1.240$ & 0.34 & 1.008 & $0.870-1.167$ & 0.916 & - & - & - \\
\hline$\geq 65$ & 0.673 & $0.467-0.970$ & 0.034 & 0.882 & $0.649-1.199$ & 0.423 & 0.871 & $0.638-1.187$ & 0.382 & - & - & - \\
\hline \multicolumn{13}{|l|}{ Race/ethnicity } \\
\hline White & I & & & I & & & - & & & I & & \\
\hline Black & 1.243 & $0.964-1.603$ & 0.093 & $1.50 \mathrm{I}$ & $1.203-1.872$ & $<0.001$ & - & - & - & 1.235 & $0.986-1.548$ & 0.066 \\
\hline Other & 0.779 & $0.540-1.125$ & 0.183 & 0.79 & $0.560-1.115$ & 0.18 & - & - & - & 0.722 & $0.510-1.021$ & 0.065 \\
\hline \multicolumn{13}{|l|}{ Grade } \\
\hline Well differentiated & I & & & 1 & & & I & & & I & & \\
\hline $\begin{array}{l}\text { Moderately } \\
\text { differentiated }\end{array}$ & 3.453 & $1.884-6.327$ & $<0.001$ & 2.6 & $1.592-4.249$ & $<0.001$ & 2.305 & $1.410-3.770$ & 0.001 & 2.312 & $1.414-3.780$ & 0.001 \\
\hline $\begin{array}{l}\text { Poorly/ } \\
\text { undifferentiated }\end{array}$ & 6.406 & $3.523-11.647$ & $<0.001$ & 4.635 & $2.859-7.512$ & $<0.001$ & 2.94 & $|.797-4.81|$ & $<0.001$ & 2.923 & $|.787-4.78|$ & 0 \\
\hline \multicolumn{13}{|l|}{ Tumor stage } \\
\hline $\mathrm{TI}$ & 1 & & & 1 & & & 1 & & & 1 & & \\
\hline $\mathrm{T} 2$ & 1.677 & $1.325-2.122$ & $<0.001$ & 1.518 & $1.227-1.878$ & $<0.001$ & 1.412 & $1.139-1.750$ & 0.002 & 1.429 & $|.154-1.77|$ & 0.001 \\
\hline T3 & 2.146 & I.669-2.758 & $<0.001$ & 1.889 & $1.502-2.375$ & $<0.001$ & 1.796 & $1.424-2.266$ & $<0.001$ & 1.808 & $1.434-2.280$ & $<0.001$ \\
\hline $\mathrm{T} 4$ & 3.739 & $2.777-5.033$ & $<0.001$ & 3.442 & $2.623-4.516$ & $<0.001$ & 2.783 & $2.115-3.662$ & $<0.001$ & 2.83 & $2.148-3.729$ & $<0.001$ \\
\hline \multicolumn{13}{|l|}{ Nodal stage } \\
\hline N2 & 1 & & & $\mathrm{I}$ & & & 1 & & & 1 & & \\
\hline N3 & 2.048 & I.764-2.377 & $<0.001$ & 2.016 & $1.754-2.318$ & $<0.001$ & $1.84 \mid$ & $1.596-2.122$ & $<0.001$ & 1.852 & $1.607-2.134$ & $<0.001$ \\
\hline \multicolumn{13}{|l|}{ ER } \\
\hline Negative & I & & & I & & & 1 & & & I & & \\
\hline Positive & 0.38 & $0.326-0.441$ & $<0.001$ & 0.388 & $0.337-0.447$ & $<0.001$ & 0.547 & $0.439-0.682$ & $<0.001$ & 0.55 & $0.44 I-0.686$ & $<0.001$ \\
\hline \multicolumn{13}{|l|}{ PR } \\
\hline Negative & I & & & I & & & 1 & & & I & & \\
\hline Positive & 0.429 & $0.370-0.498$ & $<0.001$ & 0.445 & $0.387-0.511$ & $<0.001$ & 0.773 & $0.625-0.956$ & 0.017 & 0.774 & $0.626-0.956$ & 0.018 \\
\hline \multicolumn{13}{|l|}{ Marital status } \\
\hline Married & 1 & & & 1 & & & 1 & & & 1 & & \\
\hline Unmarried & 0.771 & $0.659-0.902$ & 0.001 & 0.733 & $0.634-0.849$ & $<0.001$ & 0.768 & $0.663-0.890$ & $<0.001$ & 0.794 & $0.685-0.921$ & 0.002 \\
\hline \multicolumn{13}{|c|}{ Immediate breast reconstruction } \\
\hline No & I & & & I & & & - & & & - & & \\
\hline Yes & 1.088 & $0.937-1.262$ & 0.268 & 1.074 & $0.935-1.235$ & 0.314 & - & - & - & - & - & - \\
\hline
\end{tabular}

Abbreviations: BCSS, breast cancer-specific survival; ER, estrogen receptor; HR, hazard ratio; N, node; OS, overall survival; PR, progesterone receptor; T, tumor. 
showed that breast reconstruction significantly improved survival outcomes in unpaired patients. However, the advantage of breast reconstruction on survival disappeared in the matched group, except for patients aged $<50$ years.

Breast reconstruction is associated with better cosmetic results and quality of life. Anticipation of PMRT might lead to a delay or result in omission of reconstruction. However, a population-based study found that an increase in the use of PMRT was not associated with a decrease in breast reconstruction surgery. ${ }^{12}$ In this study, our results showed an increased trend in immediate breast reconstruction in LABC followed by PMRT, and the number of patients who received implant reconstruction was significantly higher in 2009 and 2010 compared to the period of 2004-2008. A previous SEER study also found that the national increases in PMRT receipt in patients with tumors $\leq 5 \mathrm{~cm}$ and one to three positive nodes were not accompanied by a decrease in receipt of breast reconstruction. This may represent increasing radiation oncologists' comfort by the prospect of irradiating to breast reconstruction, with improved cosmetic outcomes and quality of life for patients. ${ }^{12}$ However, the two SEER studies including ours did not include complications after breast reconstruction or whether patient implants are reconstructed as one- or two-stage events.

Several studies have found that patients who received breast implant-based reconstruction followed by PMRT had a higher rate of postoperative morbidity including reconstructive failure, surgical site infection, repeat surgery, and total complications than their autologous tissue reconstruction counterparts. ${ }^{22-24}$ This might have a significantly negative effect on patient satisfaction and cosmetic outcomes. ${ }^{25} \mathrm{~A}$ nationwide data found that implant reconstruction was on average of less cost than autologous reconstruction. However, implant reconstruction was associated with a significantly higher rate of reconstruction failure than autologous reconstruction of the irradiated breast ( $32 \%$ vs $5 \%$ ), and the cumulative cost was six times more among implant reconstruction group compared to patients who underwent autologous methods because of the significant difference in the respective reconstruction failure rates. ${ }^{6}$ It is worth noting that delayed implant reconstruction may minimize certain complications compared to immediate implant reconstruction. ${ }^{26}$ Therefore, as the frequency of immediate breast reconstruction in breast cancer continues to increase, even in the setting of PMRT, a delay in implant reconstruction or autologous tissue reconstruction may be an optimal reconstructive choice. In addition, administration of radiotherapy before mastectomy may avoid sequencing difficulties in patients suitable for breast reconstruction..$^{27,28}$

Although recent studies have indicated that immediate breast reconstruction is oncologically safe, this form of treatment for $\mathrm{LABC}$ remains controversial because of the higher rates of adverse events after administration of PMRT. ${ }^{3-9} \mathrm{~A}$ study from Hsieh et al included 192 patients with LABC and $62 \%$ of these patients received PMRT, with the results showing that breast reconstruction was an independent predictor for survival. The breast reconstruction group had a significantly lower risk of breast cancer-related death $(P=0.019)$ compared to the group that only received mastectomy. ${ }^{16}$ However, a study by Lee et al included 492 stage II and III breast cancer patients who underwent chemotherapy followed
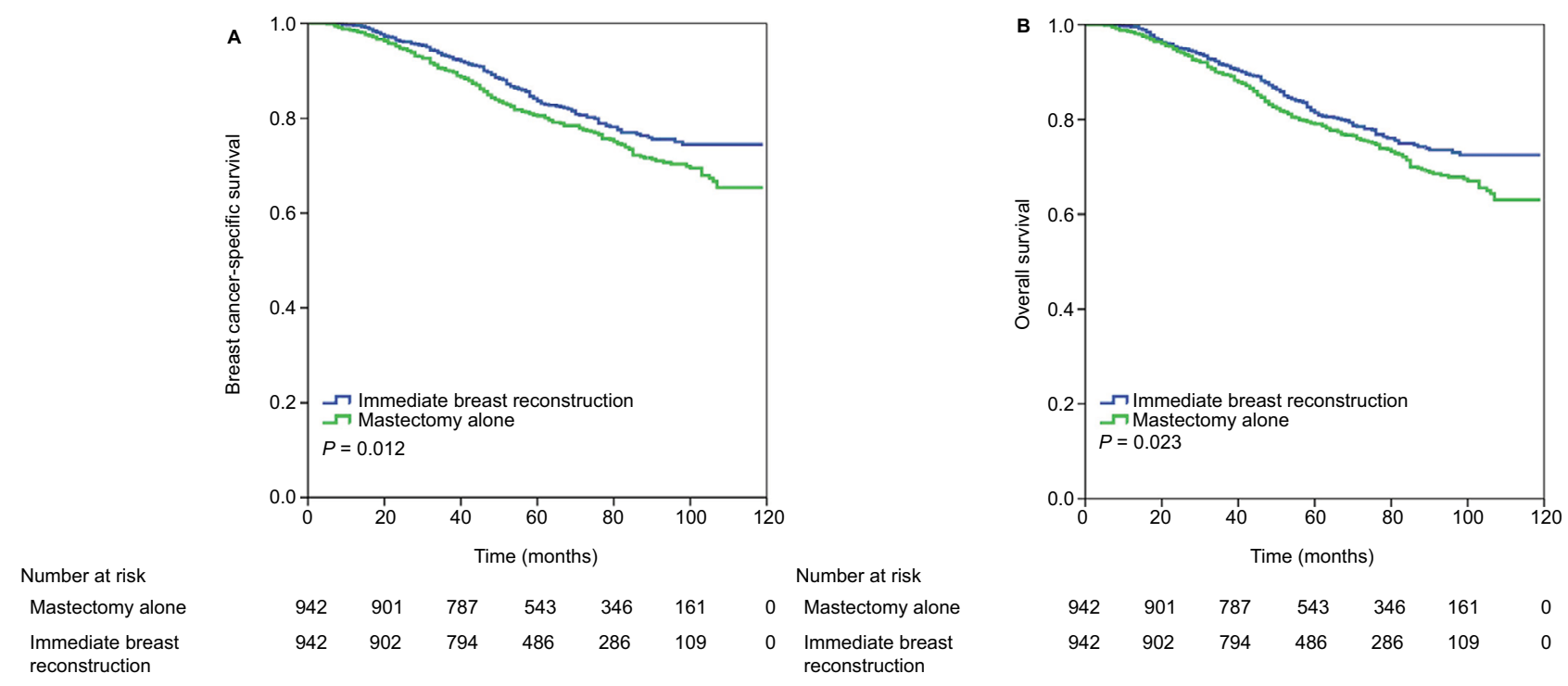

Figure 4 Survival comparison (A, breast cancer-specific survival; B, overall survival) in matched population who are $<50$ years of age with and without immediate breast reconstruction. 
Table 4 Multivariate Cox regression analysis of prognostic factors after propensity score matching in patients aged $<50$ years

\begin{tabular}{|c|c|c|c|c|c|c|}
\hline \multirow[t]{2}{*}{ Variables } & \multicolumn{3}{|l|}{ BCSS } & \multicolumn{3}{|l|}{ OS } \\
\hline & HR & $95 \% \mathrm{Cl}$ & $P$ & HR & $95 \% \mathrm{Cl}$ & $P$ \\
\hline \multicolumn{7}{|l|}{ Race/ethnicity } \\
\hline White & 1 & & & 1 & & \\
\hline Black & 1.14 & $0.834-1.557$ & $0.4 I I$ & 1.326 & $1.001-1.756$ & 0.049 \\
\hline Other & 0.562 & $0.353-0.894$ & 0.015 & 0.577 & $0.37 I-0.899$ & 0.015 \\
\hline \multicolumn{7}{|l|}{ Grade } \\
\hline Well differentiated & $\mathrm{I}$ & & & $\mathrm{I}$ & & \\
\hline Moderately differentiated & 3.786 & I.392-10.295 & 0.009 & 3.344 & $1.364-8.200$ & 0.008 \\
\hline Poorly/undifferentiated & 5.670 & $2.094-15.353$ & 0.001 & 4.867 & $|.922-11.89|$ & 0.001 \\
\hline \multicolumn{7}{|l|}{ Tumor stage } \\
\hline $\mathrm{TI}$ & $\mathrm{I}$ & & & 1 & & \\
\hline $\mathrm{T} 2$ & 2.006 & $|.432-2.8| \mid$ & $<0.001$ & $\mathrm{I} .757$ & $1.289-2.396$ & $<0.001$ \\
\hline T3 & 2.549 & $1.781-3.649$ & $<0.001$ & 2.309 & $1.659-3.212$ & $<0.001$ \\
\hline $\mathrm{T} 4$ & 4.706 & $3.104-7.133$ & $<0.001$ & 4.377 & $2.986-6.416$ & $<0.001$ \\
\hline \multicolumn{7}{|l|}{ Nodal stage } \\
\hline N2 & $\mathrm{I}$ & & & 1 & & \\
\hline N3 & 2.028 & $1.656-2.484$ & $<0.001$ & 2.019 & $1.665-2.449$ & $<0.001$ \\
\hline \multicolumn{7}{|l|}{ ER } \\
\hline Negative & $\mathrm{I}$ & & & I & & \\
\hline Positive & 0.627 & $0.454-0.866$ & 0.005 & 0.62 & $0.456-0.844$ & 0.002 \\
\hline \multicolumn{7}{|l|}{ PR } \\
\hline Negative & $\mathrm{I}$ & & & 1 & & \\
\hline Positive & 0.743 & $0.544-1.016$ & 0.063 & 0.732 & $0.543-0.986$ & 0.040 \\
\hline \multicolumn{7}{|l|}{ Marital status } \\
\hline Married & 1 & & & 1 & & \\
\hline Unmarried & 0.877 & $0.704-1.092$ & 0.240 & 0.869 & $0.706-1.070$ & 0.186 \\
\hline \multicolumn{7}{|c|}{ Immediate breast reconstruction } \\
\hline No & 1 & & & 1 & & \\
\hline Yes & 0.750 & $0.614-0.917$ & 0.005 & 0.779 & $0.644-0.942$ & 0.010 \\
\hline
\end{tabular}

Abbreviations: BCSS, breast cancer-specific survival; ER, estrogen receptor; HR, hazard ratio; N, node; OS, overall survival; PR, progesterone receptor; T, tumor.

by PMRT. The results showed that there was no statistically significant difference in local and distant recurrence, disease-free survival, and OS compared to the immediate reconstruction group. ${ }^{13}$ Similarly, Hazard et al found no difference in locoregional recurrence, disease-free status, and $O S$ in the setting of immediate breast reconstruction or mastectomy alone followed by PMRT..${ }^{14}$ Moreover, Soong et al demonstrated similar locoregional control and OS between the two groups. ${ }^{15}$

In our study, we found that patients who underwent breast reconstructions in the unmatched populations had better survival compared to mastectomy alone, which was similar to the previous SEER study assessing stage I-IV breast cancer regardless of the use of PMRT. ${ }^{29,30}$ Women who underwent breast reconstruction were more likely to be younger, be married, have a higher family income and education level, have a better overall health status, have hormone receptor-positive disease, and be diagnosed at an early stage. ${ }^{16,29-31}$ These findings were in agreement with our results. We believe that this association between breast reconstructions and survival in the unmatched population was more likely to be attributed to imbalances in socioeconomic, tumor, and treatment factors. In our study, we used the PSM method to reduce the potential selection bias in retrospective studies, and we did not find any relationship between breast reconstruction and patient survival after PSM.

Although our study lacked data on patients with local recurrence, it has been suggested that previous adipocytes may play a major role in breast cancer recurrence. Manabe et al showed that mature adipose cells can promote formation of breast carcinoma cells in a collagen gel matrix culture. ${ }^{32} \mathrm{~A}$ matched cohort study also found a higher risk of local recurrence in patients undergoing the process of lipomodeling. ${ }^{33}$ Therefore, autologous tissue reconstruction may present with poor outcome compared to the implant reconstruction group. However, a recent study indicated that immediate autologous tissue reconstruction in the setting of PMRT did not increase the occurrence of local and distant events, and 
did not decrease breast cancer-related death. ${ }^{34}$ Fertsch et al used a matching analysis, and their study found no general increased risk of recurrence between the lipomodeling and control group. ${ }^{35}$ In our study, we did not observe significant differences in survival outcomes between the two reconstruction methods. As all patients in our study received PMRT, we believe that this may have decreased the risk of locoregional recurrence. Therefore, the survival outcomes may not be significantly different in our study.

Women younger than 50 years have a higher likelihood of receiving mastectomy followed by breast reconstruction compared to older patients, ${ }^{36}$ and this was similar to our results. In addition, our subset analysis found that breast reconstruction was associated with better survival outcomes than for patients who underwent mastectomy alone and who were younger than 50 years in the matched population. The physiologic or immunologic effects in younger reconstruction patients may be the main reason for better survival in immediate breast reconstruction group as compared to patients who received mastectomy without immediate breast reconstruction. ${ }^{37}$ In the People's Republic of China, the rate of breast reconstruction is $<5 \%$, which is significantly lower than in Western countries. ${ }^{12,38}$ In addition, the median age for breast cancer is 48-50 years in the People's Republic of China compared to 64 years in the USA. In addition, mastectomy continues to account for $\sim 90 \%$ of Chinese patients. ${ }^{39}$ Therefore, our study has great significance in decision making of clinical practice for Chinese women.

Inevitably, there are several limitations in our study that should be acknowledged. First, our study was a retrospective study, which might introduce unaccounted biases. Second, the SEER database only included immediate breast reconstruction data, and patients who had undergone delayed breast reconstruction were not analyzed in our study. Some patients may have completed their reconstruction only after the follow-up time was computed. Third, we could not investigate the effect of local recurrence, cosmetic outcome, and treatment-related complications after PMRT between the two reconstruction methods. In addition, confounders such as comorbidities, body mass index, smoking, performance status, insurance type (SEER recorded the insurance status after 2007, and only 136 patients were uninsured), or clinician- and patient-related preferences can influence decision making in daily clinical practice. However, SEER database also lacks the abovementioned variables. The lack of data regarding human epidermal growth factor receptor 2 status, radiotherapy dosage, and chemotherapy regimen was another limitation.

\section{Conclusion}

In conclusion, our results suggest that there are comparable survival outcomes in LABC patients who received immediate breast reconstruction or mastectomy alone followed by PMRT, except in patients who were younger than 50 years of age. These younger patients appeared to have a survival advantage after immediate breast reconstruction. Our results demonstrate the survival outcomes in relation to immediate breast reconstruction followed by PMRT for both the physicians and the patients.

\section{Acknowledgments}

This work was partly supported by the Natural Science Foundation of Fujian Province (No. 2016J01635), the Science and Technology Planning Projects of Xiamen Science \& Technology Bureau (No. 3502Z20174070), and Guangdong Medical Research Foundation (No. A2017023).

\section{Disclosure}

The authors report no conflicts of interest in this work.

\section{References}

1. Overgaard M, Hansen PS, Overgaard J, et al. Postoperative radiotherapy in high-risk premenopausal women with breast cancer who receive adjuvant chemotherapy. Danish Breast Cancer Cooperative Group 82b Trial. N Engl J Med. 1997;337(14):949-955.

2. Katz A, Strom EA, Buchholz TA, et al. Locoregional recurrence patterns after mastectomy and doxorubicin-based chemotherapy: implications for postoperative irradiation. J Clin Oncol. 2000;18(15) 2817-2827.

3. Jagsi R, Momoh AO, Qi J, et al. Impact of radiotherapy on complications and patient-reported outcomes after breast reconstruction. $J \mathrm{Natl}$ Cancer Inst. 2018;110(2).

4. Ricci JA, Epstein S, Momoh AO, Lin SJ, Singhal D, Lee BT. A metaanalysis of implant-based breast reconstruction and timing of adjuvant radiation therapy. J Surg Res. 2017;218:108-116.

5. Chen CF, Hung CF, Lin SF, Chung YL. Does prosthesis-based breast reconstruction affect the clinical outcome of postmastectomy radiotherapy? Ann Plast Surg. 2018;80(2S Suppl 1):S7-S10.

6. Aliu O, Zhong L, Chetta MD, et al. Comparing health care resource use between implant and autologous reconstruction of the irradiated breast: a national claims-based assessment. Plast Reconstr Surg. 2017;139(6):1224e-1231e.

7. Magill LJ, Robertson FP, Jell G, Mosahebi A, Keshtgar M. Determining the outcomes of post-mastectomy radiation therapy delivered to the definitive implant in patients undergoing one- and two-stage implantbased breast reconstruction: a systematic review and meta-analysis. J Plast Reconstr Aesthet Surg. 2017;70(10):1329-1335.

8. Chuba PJ, Stefani WA, Dul C, et al. Radiation and depression associated with complications of tissue expander reconstruction. Breast Cancer Res Treat. 2017;164(3):641-647.

9. El-Sabawi B, Ho AL, Sosin M, Patel KM. Patient-centered outcomes of breast reconstruction in the setting of post-mastectomy radiotherapy: a comprehensive review of the literature. J Plast Reconstr Aesthet Surg. 2017;70(6):768-780.

10. Jagsi R, Jiang J, Momoh AO, et al. Trends and variation in use of breast reconstruction in patients with breast cancer undergoing mastectomy in the United States. J Clin Oncol. 2014;32(9):919-926. 
11. Agarwal S, Kidwell KM, Farberg A, Kozlow JH, Chung KC, Momoh AO. Immediate reconstruction of the radiated breast: recent trends contrary to traditional standards. Ann Surg Oncol. 2015;22(8):2551-2559.

12. Frasier LL, Holden S, Holden T, et al. Temporal trends in postmastectomy radiation therapy and breast reconstruction associated with changes in national comprehensive cancer network guidelines. JAMA Oncol. 2016;2(1):95-101.

13. Lee HH, Hou MF, Wei SY, et al. Comparison of long-term outcomes of postmastectomy radiotherapy between breast cancer patients with and without immediate flap reconstruction. PLoS One. 2016;11(2):e0148318.

14. Hazard L, Miercort C, Gaffney D, Leavitt D, Stewart JR. Local-regional radiation therapy after breast reconstruction: what is the appropriate target volume? A case-control study of patients treated with electron arc radiotherapy and review of the literature. Am J Clin Oncol. 2004;27(6):555-564.

15. Soong IS, Yau TK, Ho CM, et al. Post-mastectomy radiotherapy after immediate autologous breast reconstruction in primary treatment of breast cancers. Clin Oncol (R Coll Radiol). 2004;16(4):283-289.

16. Hsieh TY, Lin YN, Lin SD, et al. Immediate transverse rectus abdominis musculocutaneous flap reconstruction is associated with improved cancer-specific survival in locally advanced breast cancer. Ann Plast Surg. 2014;73(Suppl 1):S31-S36.

17. Surveillance, Epidemiology, and End Results (SEER) Program (www.seer. cancer.gov) SEER *Stat Database: Incidence - SEER 18 Regs Custom Data (with chemotherapy recode), Nov 2015 Sub (2000-2013) <Katrina/Rita Population Adjustment> - Linked To County Attributes - Total U.S., 19692014 Counties, National Cancer Institute, DCCPS, Surveillance Research Program, released July 2016, based on the November 2015 submission.

18. Agarwal J, Agarwal S, Pappas L, Neumayer L. A population-based study of breast cancer-specific survival following mastectomy and immediate or early-delayed breast reconstruction. Breast J. 2012;18(3): 226-232.

19. Wu W, Cheng S, Deng H, Wu J, Mao K, Cao M. Impact of breast cancer subtype defined by immunohistochemistry hormone receptor and HER2 status on the incidence of immediate postmastectomy reconstruction. Medicine (Baltimore). 2016;95(3):e2547.

20. Rosenbaum PR, Rubin DB. Constructing a control group using multivariate matched sampling methods that incorporate the propensity score. Am Stat. 1985,39(1):33-38.

21. Austin PC. An introduction to propensity score methods for reducing the effects of confounding in observational studies. Multivariate Behav Res. 2011;46(3):399-424.

22. Tsoi B, Ziolkowski NI, Thoma A, Campbell K, O'Reilly D, Goeree R. Safety of tissue expander/implant versus autologous abdominal tissue breast reconstruction in postmastectomy breast cancer patients: a systematic review and meta-analysis. Plast Reconstr Surg. 2014;133(2): 234-249.

23. El-Sabawi B, Sosin M, Carey JN, Nahabedian MY, Patel KM. Breast reconstruction and adjuvant therapy: a systematic review of surgical outcomes. J Surg Oncol. 2015;112(5):458-464.

24. Barry M, Kell MR. Radiotherapy and breast reconstruction: a metaanalysis. Breast Cancer Res Treat. 2011;127(1):15-22.
25. Magill LJ, Robertson FP, Jell G, Mosahebi A, Keshtgar M. Determining the outcomes of post-mastectomy radiation therapy delivered to the definitive implant in patients undergoing one- and two-stage implantbased breast reconstruction: a systematic review and meta-analysis. $J$ Plast Reconstr Aesthet Surg. 2017;70(10):1329-1335.

26. Seth AK, Silver HR, Hirsch EM, Kim JY, Fine NA. Comparison of delayed and immediate tissue expander breast reconstruction in the setting of postmastectomy radiation therapy. Ann Plast Surg. 2015;75(5):503-507.

27. Tansley P, Ramsey K, Wong S, Guerrieri M, Pitcher M, Grinsell D. New treatment sequence protocol to reconstruct locally advanced breast cancer. ANZ J Surg. 2013;83(9):630-635.

28. Paillocher N, Florczak AS, Richard M, et al. Evaluation of mastectomy with immediate autologous latissimus dorsi breast reconstruction following neoadjuvant chemotherapy and radiation therapy: a single institution study of 111 cases of invasive breast carcinoma. Eur J Surg Oncol. 2016;42(7):949-955.

29. Bezuhly M, Temple C, Sigurdson LJ, Davis RB, Flowerdew G, Cook EF Jr. Immediate postmastectomy reconstruction is associated with improved breast cancer-specific survival: evidence and new challenges from the Surveillance, epidemiology, and end results database. Cancer. 2009;115(20):4648-4654.

30. Jiang YZ, Liu YR, Yu KD, Zuo WJ, Shao ZM. Immediate postmastectomy breast reconstruction showed limited advantage in patient survival after stratifying by family income. PLoS One. 2013;8(12):e82807.

31. Agarwal S, Liu JH, Crisera CA, Buys S, Agarwal JP. Survival in breast cancer patients undergoing immediate breast reconstruction. Breast $J$. 2010;16(5):503-509.

32. Manabe Y, Toda S, Miyazaki K, Sugihara H. Mature adipocytes, but not preadipocytes, promote the growth of breast carcinoma cells in collagen gel matrix culture through cancer-stromal cell interactions. J Pathol. 2003;201(2):221-228.

33. Petit JY, Rietjens M, Botteri E, et al. Evaluation of fat grafting safety in patients with intraepithelial neoplasia: a matched-cohort study. Ann Oncol. 2013;24(6):1479-1484.

34. Maalouf C, Bou-Merhi J, Karam E, Patocskai E, Danino AM. The impact of autologous breast reconstruction using DIEP flap on the oncologic efficacy of radiation therapy. Ann Chir Plast Esthet. 2017;62(6):630-636.

35. Fertsch S, Hagouan M, Munder B, et al. Increased risk of recurrence associated with certain risk factors in breast cancer patients after DIEPflap reconstruction and lipofilling - a matched cohort study with 200 patients. Gland Surg. 2017;6(4):315-323.

36. Morrow M, Scott SK, Menck HR, Mustoe TA, Winchester DP. Factors influencing the use of breast reconstruction postmastectomy: a National Cancer Database study. J Am Coll Surg. 2001;192(1):1-8.

37. Hoshaw SJ, Klein PJ, Clark BD, Cook RR, Perkins LL. Breast implants and cancer: causation, delayed detection, and survival. Plast Reconstr Surg. 2001;107(6):1393-1407.

38. Chen Y, Chen J, Chen J, et al. [Current trends of breast reconstruction after mastectomy for breast cancer patients in China: a survey report]. Zhonghua Zhong Liu Za Zhi. 2014;36(11):851-857. Chinese

39. Fan L, Strasser-Weippl K, Li JJ, et al. Breast cancer in China. Lancet Oncol. 2014;15(7):e279-e289.
Cancer Management and Research

\section{Publish your work in this journal}

Cancer Management and Research is an international, peer-reviewed open access journal focusing on cancer research and the optimal use of preventative and integrated treatment interventions to achieve improved outcomes, enhanced survival and quality of life for the cancer patient. The manuscript management system is completely online and includes

\section{Dovepress}

a very quick and fair peer-review system, which is all easy to use. Visit http://www.dovepress.com/testimonials.php to read real quotes from published authors. 